\title{
Lactobacillus acidophilus as a live vehicle for oral immunization against chicken anemia virus.
}

\begin{abstract}
The AcmA binding domains of Lactococcus lactis were used to display the VP1 protein of chicken anemia virus (CAV) on Lactobacillus acidophilus. One and two repeats of the cell wall binding domain of acmA gene were amplified from L. lactis MG1363 genome and then inserted into co-expression vector, pBudCE4.1. The VP1 gene of CAV was then fused to the acmA sequences and the VP2 gene was cloned into the second MCS of the same vector before transformation into Escherichia coli. The expressed recombinant proteins were purified using a His-tag affinity column and mixed with a culture of L. acidophilus. Whole cell ELISA and immunofluorescence assay showed the binding of the recombinant VP1 protein on the surface of the bacterial cells. The lactobacilli cells carrying the CAV VP1 protein were used to immunize specific pathogen-free chickens through the oral route. A moderate level of neutralizing antibody to CAV was detected in the serum of the immunized chickens. A VP1-specific proliferative response was observed in splenocytes of the chickens after oral immunization. The vaccinated groups also showed increased levels of Th1 cytokines interleukin (IL)-2, IL-12, and IFN- $\gamma$. These observations suggest that L. acidophilus can be used in the delivery of vaccines to chickens.
\end{abstract}

Keyword: Chicken anemia virus; Oral vaccine; AcmA anchor protein; Surface display; Lactobacillus acidophilus. 